\title{
Multiscale analysis of thermography imaging dynamic for sol-gel coating discrimination
}

\author{
S. Mezghani*, E. Perrin*, J.L Bodnar**, B. Cauwe ${ }^{* * *}$, V. Vrabie* \\ ${ }^{*}$ CReSTIC/Châlons, Université de Reims Champagne-Ardenne, Chaussée du port, 51000 Châlons-en- \\ Champagne, France \\ ** GRESPI/ECATHERM, Université de Reims Champagne-Ardenne, BP 1039, 51687 Reims, France \\ ${ }^{* * *}$ CRITT-MDTS, 3 Boulevard Jean Delautre - ZHT du Moulin Leblanc, 08000 Charleville-Mézières, France
}

\begin{abstract}
Infrared thermography is a non-destructive method that is becoming attractive due to its ability to inspect noninvasively large areas in short times. This work demonstrates that the analysis of the thermographic response of coating layer can be greatly improved with a multiscale decomposition. Based on 2D discrete wavelets transform, effective scales in the thermographic responses of samples can be selected. Then, the dynamic thermal response was analyzed using cross-correlation measure. Results show that thermal transient images obtained with this decomposition, during both excitation and cooling step, provide important information for detecting the presence of the sol-gel coating.
\end{abstract}

\section{Introduction}

Non-destructive testing (NDT) is becoming an inseparable part of modern engineering applications. Increasing design complexity, the use of new materials and high requirements for reliability of structures require continuous development of testing methods for detection and evaluation of structural defects. A number of different testing methods have been developed for damage detection including visual inspection, passive and active approaches based on ultrasonic signals, liquid penetrant testing, radiographic methods, etc. [1]. Infrared Thermography testing has found widespread use in non-contact, non-intrusive testing [2]. Pulsed Phase Thermography (PPT) approach was introduced for non-destructive evaluation in infrared thermography applications as an interesting technique [3]. Measured signals (heating and/or decay) as a time response, governed by physical processes (thermal conductivity, thermal diffusivity, etc.), are generally modeled in order to extract different parameters and thus to characterize the thermographic response of coatings [4]. From a signal processing point of view, multi-resolution techniques such as the wavelet transform have been successfully applied to engineering problems, financial time series studies, etc. because of its powerful feature extraction capability. Such technique can be used to select effective scale in the thermographic response of samples. Indeed, in the range of wavelength, each thermal image is a layer in the multilayer image. At each instant, the multiscale decomposition of a recorded thermal image can be used to identify and select the best scale corresponding to the coating response and not to environmental, substrate, or other factors that affect the thermographic response of a sample. The advanced signal processing tools, chosen in this paper is the discrete wavelets transform (DWT). It can be viewed as an adaptive filtering tool that improves the analysis of raw acquired thermographic responses. In order to estimate the degree of similarity between thermal images and thus to assess the effectiveness of the multiscale decomposition, we have chosen a cross correlation measure. From an application point of view, two kinds of samples have been analysed: uncoated and sol-gel coated metal plate samples painted with a commercial white paint. The proposed signal processing tool is also expected to demonstrate the behavior of sol-gel thermal barrier coatings.

\section{Materials and methods}

\subsection{Samples}

Ten rectangular steel plate samples of $6 \mathrm{~cm}$ wide, $2 \mathrm{~cm}$ long and $9.4 \mathrm{~mm}$ thick were used. Five samples were covered by thin film of sol gel of almost $12 \mu \mathrm{m}$ thick. The five uncoated and the five sol-gel coated meal plate samples were next painted with a commercial white paint. Figure 1 shows cross-section micrographs of an uncoated painted sample and a sol-gel coated painted sample obtained by optical microscopy (Olympus BX60). 


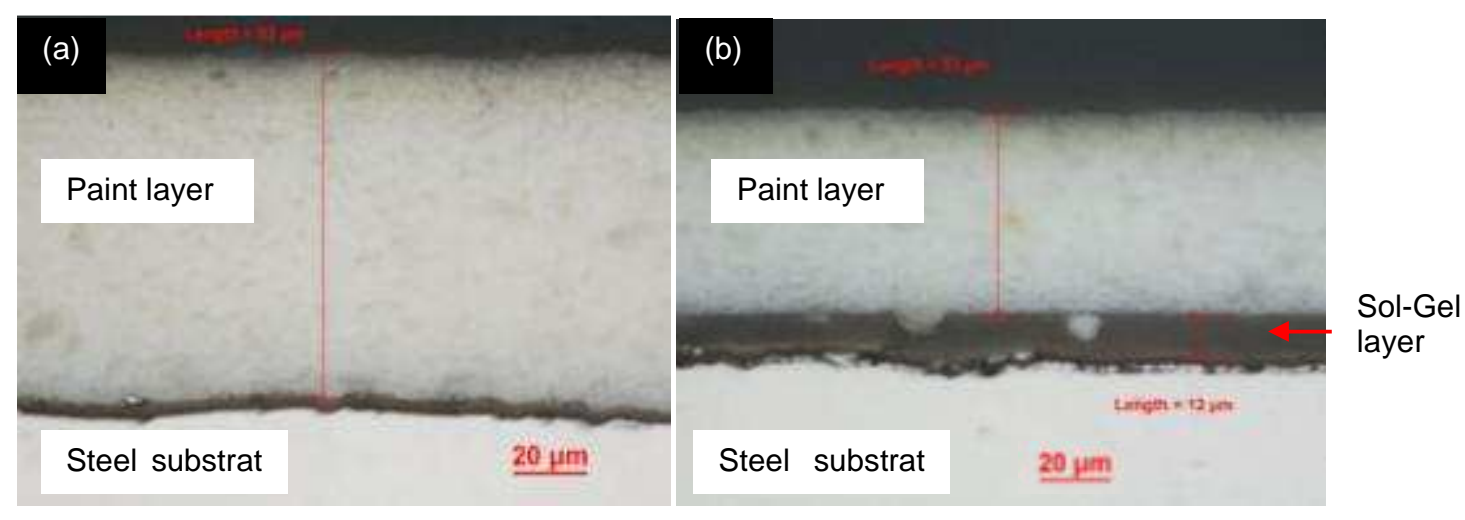

Fig. 1. Cross section micrographs of (a) an uncoated painted sample and (b) a sol-gel coated painted sample.

\subsection{Acquisition system}

The experimental system consists of a pulsed thermography bench [5]. This system, designed in the GRESPI physical laboratory, is shown in Figure 2-b.The hardware consists of an IR camera (manufacturer FLIRSC655, Infrared imaging video bolometer (IRVB) with long wave Infrared; sensor pixel size is $100 \mu \mathrm{m}$; frame rate $f_{\text {rate }}=200 \mathrm{~Hz}$ ). The sample surface is submitted to a heat pulse using an external heat flux excitation source (electrical power of $2 \mathrm{~kW}$, the duration pulse of one second and total recording time of ten seconds). The heating source is placed at the distance of $70 \mathrm{~cm}$ from the sample and surrounded by a box in order to focus energy on the sample (Fig 2-b). All ten samples were fixed on the same support (Fig 2-a). Excitation is sequentially applied on each line hiding the other samples by an insulator. A standard PC controls the IR camera, acquires and processes the image dataset.
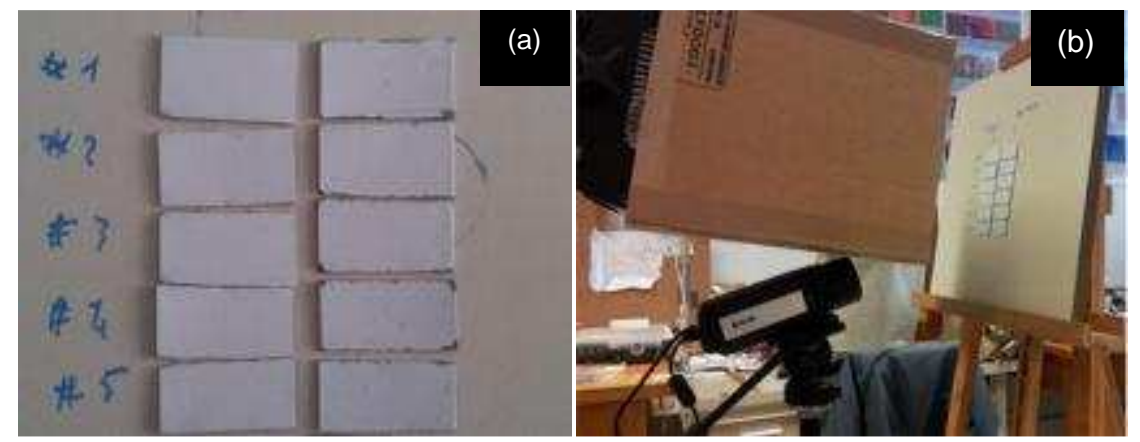

Fig. 2. (a) Samples: uncoated samples are on the left column and the sol-gel coated samples on the right column. (b) Experimental thermographic bench using a Flash lamp and a FLIRß placed at the distance of 70 $\mathrm{cm}$ from the sample and connected to a PC computer for recording the thermographic responses of samples to pulse heating excitations.

\subsection{Recorded dataset}

Each thermal image corresponds to a two-dimensional data defined by the distribution of the thermographic response on the sample at the acquisition time $t$. The temporal image sequence is stored into a 3D data structure as shown in Fig 3.a. Data cubes are constructed by stacking up the temporal sequences of thermal images:

$$
A=\left\{\mathrm{A}\left(\mathrm{x}, \mathrm{y}, \mathrm{t}_{\mathrm{k}}\right) \mid x \in\left[1, N_{x}\right], y \in\left[1, N_{y}\right], t_{k} \in[0, T]\right\}
$$

Where $x$ and $y$ are the coordinates in the $X-Y$ plane of the thermal image of size $N_{x} \times N_{y}$, with $N_{x}=320 \mathrm{px}$ and $N_{y}=120 \mathrm{px}$ in our case, " $t_{k}$ " is the acquisition time that varies between 0 and $T=N_{t} / f_{\text {rate }}$ seconds, with $N_{t}$ the total number of acquired images and $f_{\text {rate }}$ is the acquisition frequency $(200 \mathrm{~Hz}) . T$ is fixed here to ten seconds.

Figures 3-b and 3-c show the raw thermal images acquired at $t_{k}=3 s$, that is, the matrix $\mathbf{A}_{k}(x, y)=\mathrm{A}\left(\mathrm{x}, \mathrm{y}, \mathrm{t}_{\mathrm{k}}\right)$ for the uncoated (b) and sol-gel coated (c) samples that have been presented in Figure 1. These raw thermal images do 
not allow discriminating the sol-gel coating effect between two samples. Thermal discrimination threshold are relatively uncommon.

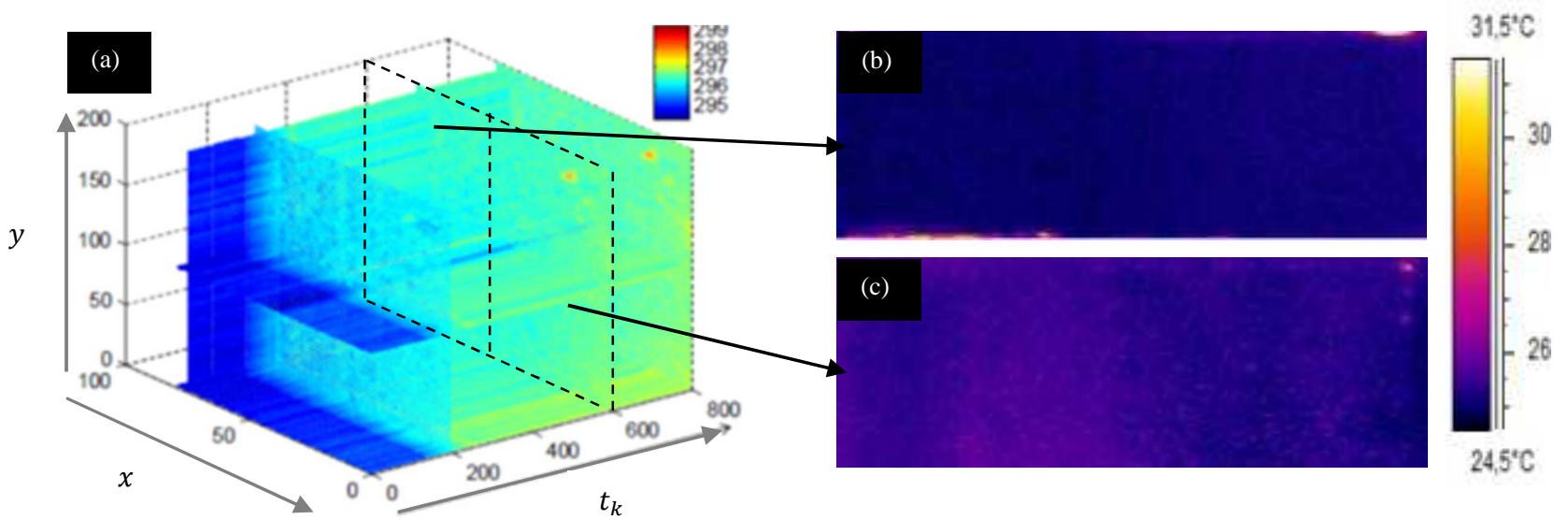

Fig. 3. (a) $3 D$ thermal data recorded by the FLIR camera on one sample; (b-c) Thermal images $\mathbf{A}_{k}$ recorded at $t_{k}=3$ s on two samples: uncoated (b) and sol-gel coated (c)

In this paper we propose to use a multiscale decomposition based on wavelets transform as a new concept of thermal image filtering for enhancing coating effect discrimination.

\section{Discrete wavelets decomposition}

At each instant $t_{k}$, the discrete wavelets decomposition is used as a multiscale analysis of the acquired thermographic image $\mathbf{A}_{k}$ at different scales. Each $\mathbf{A}_{k}$ image is processed through a series of high-pass and low-pass filters to analyze the high "HF" and low "LF" frequencies. The wavelet filtering function is a two variable scaling function, denoted $\phi(x, y)$. This function is iteratively applied on the approximate coefficients " $C A_{k}^{j}$ " at the scale level $j$ in order to identify the down-sampled output, i.e. the approximate coefficients " $C A_{k}^{j+1}$ at the next scale level $j+1$ :

$$
C A_{k}^{j+1}\left(n_{1}, n_{2}\right)=\sum_{x=1}^{N_{x}} \sum_{y=1}^{N_{y}} \phi(x, y) C A_{k}^{j}\left(2 n_{1}-x, 2 n_{2}-y\right),
$$

With the initialisation " $C A_{k}^{0}=\mathbf{A}_{k}$ ", that is, the thermographic image.

At each scale $j$, we can also compute the detail coefficients " $C D_{k}^{j}$ " [6] as following:

$$
C D_{k}^{j}\left(n_{1}, n_{2}\right)=C A_{k}^{j-1}\left(n_{1}, n_{2}\right)-C A_{k}^{j}\left(n_{1}, n_{2}\right)
$$

When $j=1$, the detail coefficients are the difference between the thermographic image $\mathbf{A}_{k}\left(n_{1}, n_{2}\right)$ and the approximate coefficients " $C A_{k}^{1}$ " at the scale level $j=1$.

Then, the coefficients can be used to reconstruct the details $D_{k}^{j}$ at each scale level $j$ of the thermographic image $\mathbf{A}_{k}$ by employing the inverse discrete wavelet transform (IDWT).

There are different wavelets basis functions but there is no well-defined rule for selecting a specific wavelet function $\phi(x, y)$ for a given application [6]. For time series applications, the time and frequency localization properties are the more common criteria to choose one more suitable wavelet than another [7]. For image filtering, criteria are given by spatial frequencies properties. In this study, the "daubechies 4" (db4) wavelet scaling function was selected as it has a compact support. The wavelet toolbox from Matlab (R2010b) was used to conduct the discrete wavelets decomposition [6].

Figure 4 shows an example of multiscale level decomposition of a raw thermal image $\mathbf{A}_{k}$ using the discrete wavelet function $\mathrm{db} 4$ on seven levels. In accordance to the theory presented in [8], the seventh level wavelet decomposition was selected since the frequency sampling used in this study was $200 \mathrm{kHz}$. $D_{k}^{1}$ to $D_{k}^{7}$ represent the filtered image of the details from the level one to level seven (shown in order from top to bottom). 


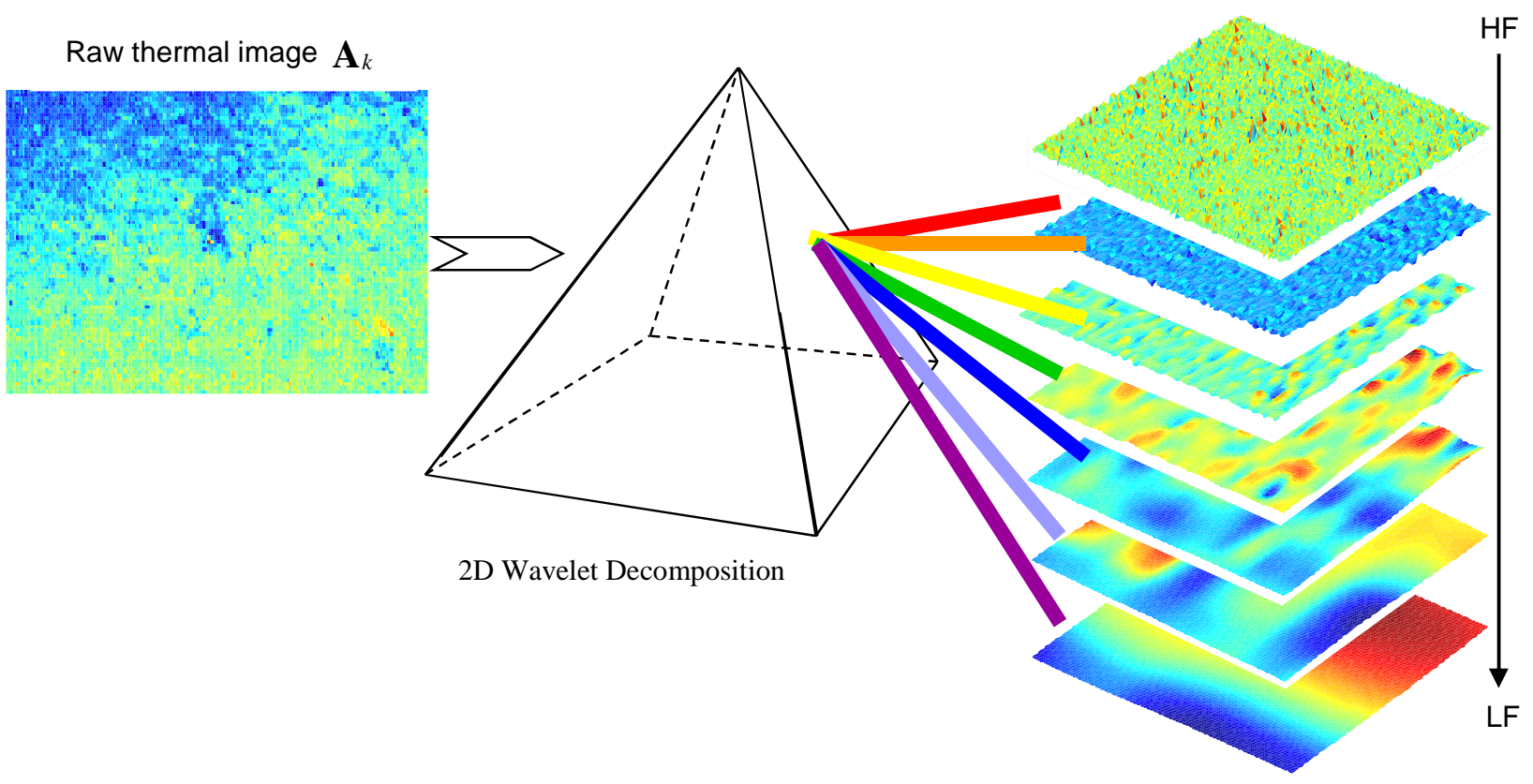

Fig. 4. Example of the multiscale level decomposition of a raw thermal $A_{k}$ using the discrete wavelet decomposition "daubechies 4" on seven levels. The details of this thermal image, $D_{k}^{1}$ to $D_{k}^{7}$, are represented from top to bottom.

\section{Cross correlation}

The principle of the correlation of images was chosen to quantify the behavior of uncoated and sol-gel coated samples during thermal excitation and cooling phases test. The correlation coefficient estimates the degree to which two thermal images are correlated. Thus, to quantify the variation of the thermal flows response of the samples, we compute at each instant $t_{k}$ the cross-correlation coefficient " $r\left(t_{k}\right)$ ":

$$
r\left(t_{k}\right)=\frac{\sum_{x, y}\left(\mathbf{A}_{k}(x, y)-\overline{\mathbf{A}}_{k}\right)-\left(\mathbf{A}_{0}(x, y)-\overline{\mathbf{A}}_{0}\right)}{\sqrt{\sum_{x, y}\left(\mathbf{A}_{k}(x, y)-\overline{\mathbf{A}}_{k}\right)^{2}} \sqrt{\sum_{x, y}\left(\mathbf{A}_{0}(x, y)-\overline{\mathbf{A}}_{0}\right)^{2}}},
$$

between the reference thermal image $\mathbf{A}_{0}$ acquired before the heat excitation $\left(t_{k}=0 s\right)$ and the thermal image $\mathbf{A}_{k}$ acquired at the instant $t_{k}$, where $\overline{A_{k}}$ is the mean of $A_{k}$ and $\overline{A_{0}}$ is the mean of $A_{0}$. When applying the multiscale decomposition, the cross-correlation coefficient can be computed at each scale $j$ by replacing in the expression above the thermal image $A_{k}$ with its detail $D_{k}^{j}$ and $A_{0}$ with the detail $D_{0}^{j}$ of the reference thermal image.

This method was applied both on raw thermographic images and multiscale decomposition of images to select the most effective wavelength band related to the sample thermal response.

\section{Results and discussion}

Table 1 shows an example of the multiscale decomposition on seven levels $\left(D_{k}^{1}\right.$ to $D_{k}^{7}$,) of thermal images that have been acquired at the acquisition time $t_{k}=3 \mathrm{~s}$ on an uncoated (right column) and a sol gel coated (left column). The raw images are shown in the first line. The details show the thermal information at different scales from high frequency to low frequency from $D_{k}^{1}$ to $D_{k}^{7}$.

The visual inspection can be summarized by the following:

- $\quad$ For the first three levels $\mathrm{D}_{\mathrm{k}}^{1}, \mathrm{D}_{\mathrm{k}}^{2}$, and $\mathrm{D}_{\mathrm{k}}^{3}$, any difference between uncoated and sol-gel coated samples can be observed at these levels. These scales can be interpreted as representing the surface roughness or noise signature of the sample and they are independent of thermal excitation. 
Table 1. Comparison of multiscale thermal images components $\left(D_{k}^{1}\right.$ to $D_{k}^{7}$,) of sol gel coated (left) and uncoated sample (right) based on the wavelets decomposition with "db4" wavelets. The first line shows row images (first line) and corresponding detail component with seven increasing level from top to bottom.

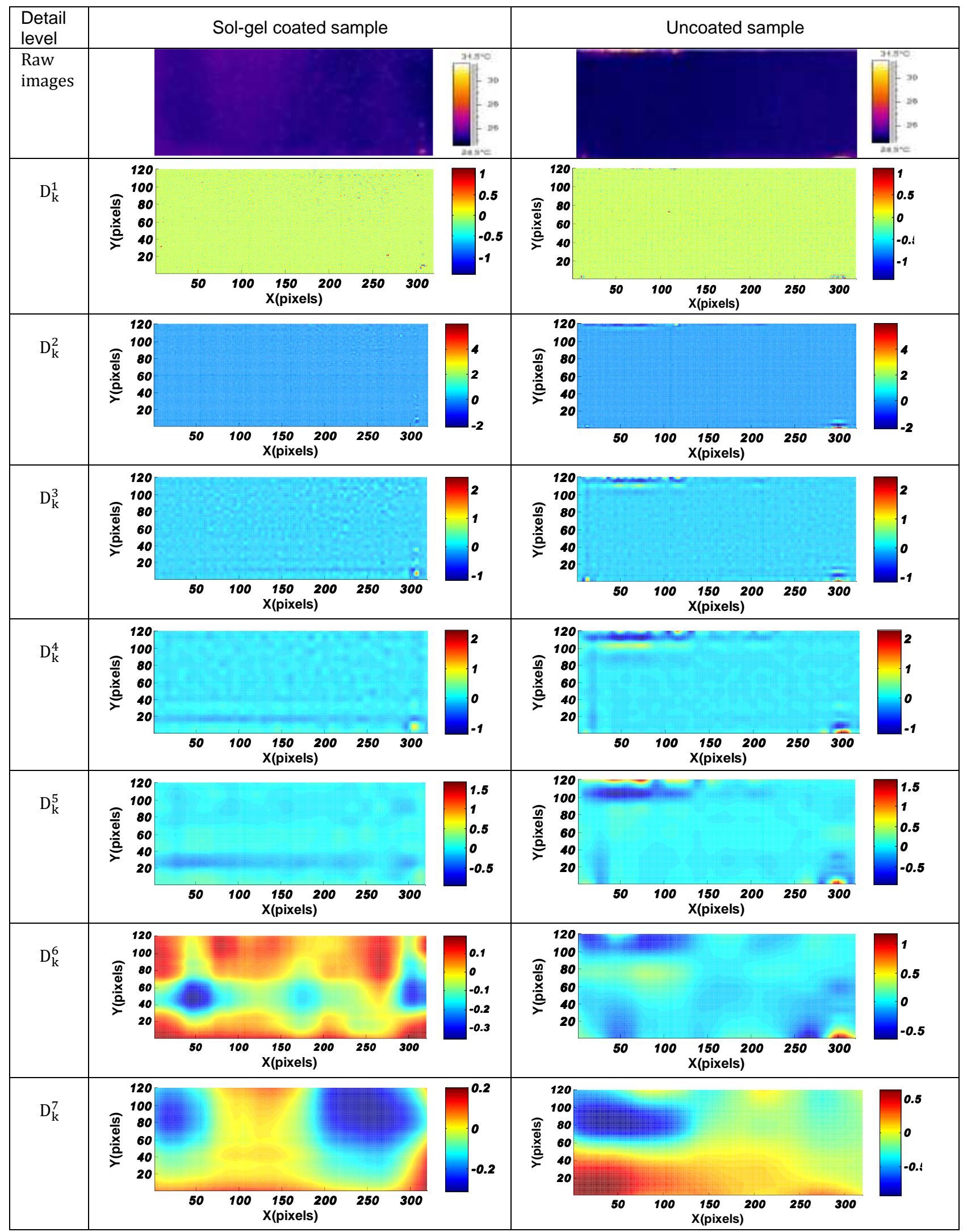


- The fourth level details to six levels of details $\left(\mathrm{D}_{\mathrm{k}}^{4}\right.$ to $\left.\mathrm{D}_{\mathrm{k}}^{6}\right)$ highlight a great difference on thermal images amplitudes between uncoated and sol-gel coated samples. These scales can be influenced by both paint and sol-gel coating layers thicknesses.

- The last detail leve/ $\mathrm{D}_{\mathrm{k}}^{7}$ : As observed in Table1, this last detail level which represents macro-scale informations (low frequency), can be related to the surface shape of the substrate layer.

Table 2 shows the time evolution of the cross-correlation coefficients during excitation and cooling period for all thermal images acquired from 0 to $3 s$ and for their seventh scale details levels with "db4" wavelet on uncoated (red plot) and solgel coated (blue plot) samples.

Table 2. Time evolution of the cross correlation coefficients during excitation $(0 s-1 s)$ and cooling $(1 s-3 s)$ period for (a) raw thermal image and (b) to ( $h$ ) for their seventh scale details levels with "db4" wavelet on uncoated (red plot) and sol-gel coated samples (blue plot).

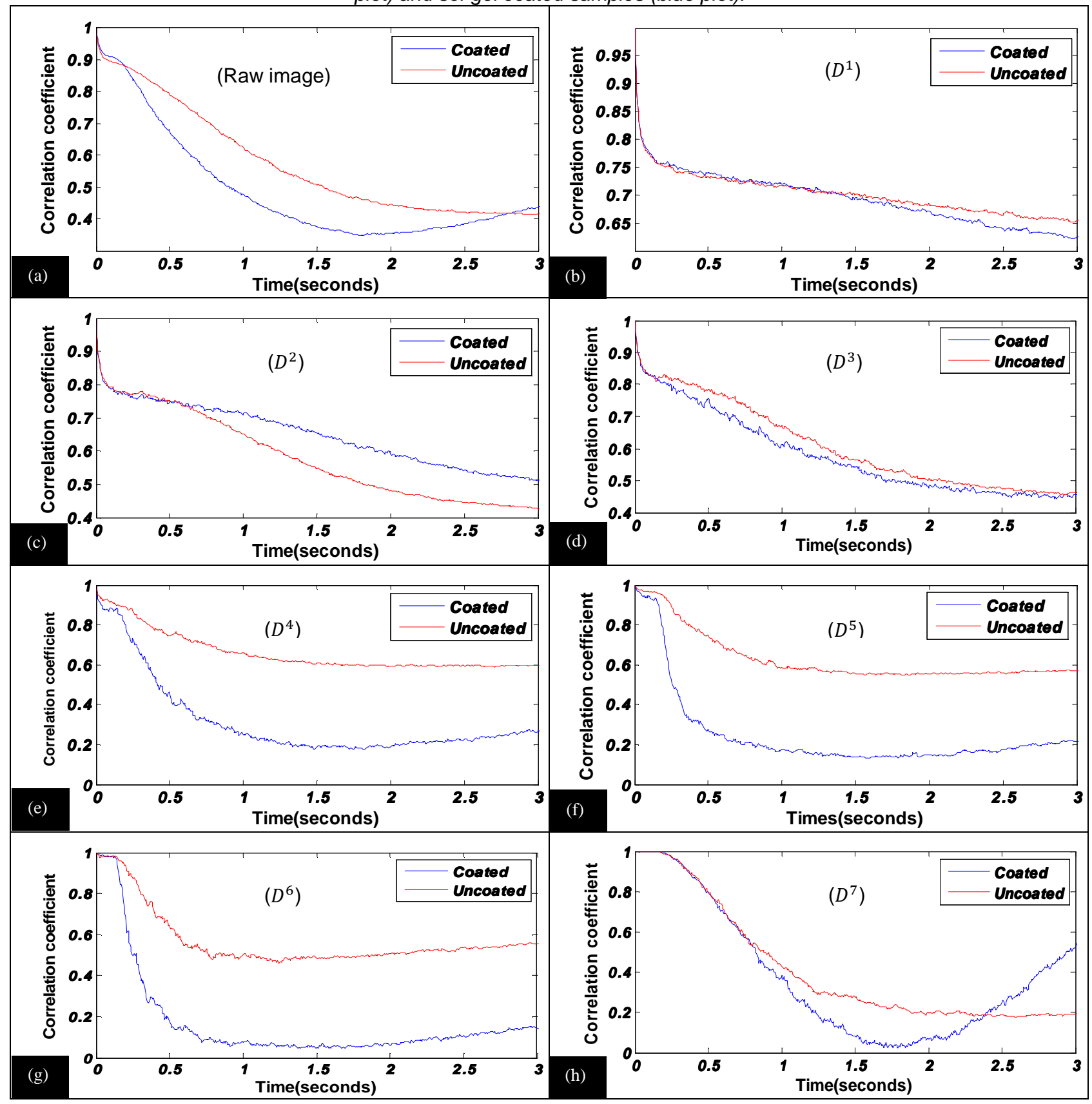


We can see that sol-gel coated samples appeared to warm up at a faster rate during the excitation heating phase than uncoated samples from detail level $\mathrm{D}_{\mathrm{k}}^{4}$ to $\mathrm{D}_{\mathrm{k}}^{6}$. The cross-correlation evolution determined at the different scales of the thermal images showed good agreement for the selected multiscale decomposition of thermal image (Table 1.e to 1.g).

In order to validate this result, a discrimination rate parameter were used to complementary quantify the discrimination between coated and uncoated samples from theirs cross correlation curves. This information is critical to assess this robustness of both multiscale decomposition and cross correlation. It's given by the following equation:

$$
d r^{j}=1-\frac{\sum_{t_{k}=0}^{T} r_{\text {uncoated }}^{j}\left(t_{k}\right)}{\sum_{t_{k}=0}^{T} r_{\text {solgel-coated }}^{j}\left(t_{k}\right)}
$$

where $j$ represents the detail level for $j=1, \ldots 7$ or the raw image for $j=0$.

This parameter represents the relative deviation between the area of cross correlation curves of uncoated and coated samples. Table 3 shows the discrimination rate between the correlation curves of different scales and of raw thermal images.

Table 3. Discrimination rates of thermal response of sol gel coated and an uncoated sample for raw thermal images and multiscale wavelet filtered components using Daubechies "db4" wavelet function, at seven levels.

\begin{tabular}{|c|c|c|c|c|c|c|c|c|}
\hline Analyzed image & Raw & $\mathrm{D}^{1}$ & $\mathrm{D}^{2}$ & $\mathrm{D}^{3}$ & $\mathrm{D}^{4}$ & $\mathrm{D}^{5}$ & $\mathrm{D}^{6}$ & $\mathrm{D} 7$ \\
\hline Discrimination rate $d r^{j}(\%)$ & 14.47 & 1.2 & -10.48 & 4.76 & 52.18 & 60.88 & 70.14 & 6.65 \\
\hline
\end{tabular}

The results show that wavelets decomposition allows an enhancing discrimination of thermographic responses between coated and uncoated samples by selecting details levels better corresponding to the thermal signature of the solgel coating. Hence, the choice of an optimal level may improve the discrimination of thermographic response, as well as the possibility of matching several levels of details.

\section{Conclusion}

Pulsed Phase Thermography method can successfully be used for non-destructive evaluation in infrared thermography. To evaluate the thermal behavior of the coating, processing of thermal image dynamic using a cross correlation tool coupled to a multiscale decomposition of thermal response using DWT was proposed. We have shown that the wavelet multiscale decomposition can improve the analysis of recorded thermal images. Indeed, the multiscale decomposition in seven levels using "db4" allows to identify the effective levels discriminating coated and uncoated samples $\left(D^{4}-D^{6}\right)$ levels according to the experimental conditions of this study.

\section{Acknowledgement}

The research for this paper was financially supported by Communauté d'agglomération de Châlons-en-Champagne, France.

\section{REFERENCES}

[1] Jakub R.,Lukasz Lukasz .P, Szwedo M.,T. Uhl, W. J. Staszewski "Thermography of Metallic and Composite Structures - review of applications ", International Workshop on smart materials, structures \& SHM NDT, Canada, 2013.

[2] Sultan R., Guirguis S., Younes M., El-Soaly E., "Active infrared thermography technique for the non destructive testing of composite material", International Journal of Mechanical Engineering and Robotics Research, 1(3), pp. 131-142, 2012.

[3] Maldague X.P, Galmiche F., Ziadi A., "Advances in Pulsed Phase Thermography", Physics \& Technology, 43, pp. 175-181, 2002.

[4] G. Mayr, B. Plank, J. Sekelja, G. Hendorfer, "Active thermography as a quantitative method for non-destructive evaluation of porous carbon fiber reinforced polymers", NDT\&E International 44, pp. 537-543, 2011. 
[5] Vrabie V., Perrin E., Bodnar J-L., Mouhoubi K., and Detalle V. "Active IR thermography processing based on higher order statistics for non-destructive evaluation", 20th European Signal Processing Conference Bucharest, Romania, 27-31 August 2012.

[6] Chowdhury SK., Nimbarte AD., Jaridi M., Creese RC., "Discrete wavelet transform analysis of surface electromyography for the fatigue assessment of neck and shoulder muscles", Journal of Electromyography and Kinesiology, 23 (5), pp. 995-1003, 2013.

[7] Mezghani S., Sabri L., El Mansori M., Zahouani H., "On the optimal choice of wavelet function for multiscale honed surface characterization", Journal of Physics: Conference Series vol. 311, issue 1, 19 August 2011.

[8] Mohd Tumari Z., Sudirman R., Ahmad A. H "Selection of a Suitable Wavelet for Cognitive Memory Using Electroencephalograph Signal" S, Scientific research Engineering, 5(5B), pp. 15-19, 2013. 